\title{
The effects of fan and door opening on a cold storage room: a numerical study
}

\author{
Azada Ayarmal Ole Melhus Arnab Chaudhuri \\ Department of Civil Engineering and Energy Technology \\ OsloMet - Oslo Metropolitan University, Oslo, Norway. \\ azada@me.com, ole.melhus@oslomet.no, arnab.chaudhuri@oslomet.no
}

\begin{abstract}
We present numerical analysis of a cold storage room with a product load, a fan, and a sliding door by solving compressible form of the conservative 3D NavierStokes equation (without Boussinesq approximation) together with the energy and mass fraction equations. The commercial computer program StarCCM+ is used for this purpose. The sliding door is treated with an overset mesh on a suitable background mesh. A rigid body translation is applied by a field-function to model the opening and closing of the door. The preliminary study captures essential features of the effect of the energy transport and the cooling of the product load by the fan and the hot, moist air exchange through the doorway. The airflow behavior and heat/mass transfer mechanisms are studied and presented for several numerical experiments.

Keywords: Cold storage room, Natural convection, Forced convection, Numerical simulations
\end{abstract}

\section{Introduction}

Cold storage rooms are designed for preservation of perishable commodities at low temperatures and are widely used in food/pharmaceutical/photographic industries. Multiple coupled interactions, involving heat-massmomentum transfer and phase change of constituent components play important roles during the operating condition of a cold store in such applications. Temperature and moisture control are the key parameters for optimal operation and energy consumption. Numerous guidelines are available for successful design and implementation for cold storage rooms. Among several other factors, infiltration of hot/moist air through open doors may become crucial for the performance of the rooms (infiltration load). Study of air infiltration is also very important for analyzing indoor air pollutant behavior, smoke control, design of clean room, hospital operating theatres, paint booths, restaurant kitchen etc.

In their study, (Wilson and Kiel, 1990) made experimental measurements in density driven counterflow through an open door. With a simple quasi-steady inviscid model, they provided a set of practical equations for estimation of the flow through an open door in a sealed room. A doorway opening possibly can result in trans- port of infectious air out of the isolation room, leading to a breakdown in isolation conditions (Tang et al., 2005) in negative-pressure isolation room. In this context, the study of different types of doors (variable speed single and double, sliding and hinged doors) with moving human figure are presented in (Tang et al., 2013) by a 1:10 scale water tank. They reported that double-hinged doors are associated with the greatest risk of leakage into or out of the room. The decreasing order risk of leakage reported as follows: double-hinged $>$ single-hinged $>$ double sliding $>$ single sliding doors. Apart from experimental approach, computational fluid dynamics tools are more cost effective alternatives to study the fundamental processes involved during the operation of a cold storage room. Nevertheless, resolving complex coupled interactions via realistic numerical models is also challenging. Large-eddy simulation (LES) is performed by (Choi and Edwards, 2012) to study the human-induced contaminant transport in roomcompartments. The effects of human and door motions and vent system activity in vestibule connected compartments (contaminated and clean room) are reported. According to their study, swinging door motion induces up to six times more compartment-to-compartment transport than sliding door motion. The human wake effect enhances the compartment-to-compartment transport. Experiments and unsteady Computational Fluid Dynamics (CFD) simulations are presented by (Chang et al., 2016) to investigate the contaminant inleakage produced by the hinged door opening and closing. The indoor air flow and mass exchange induced by the rotating motion of a hinged door separating two rooms is investigated by (Papakonstantis et al., 2017).

Experimental studies of air flow, temperature and humidity patterns in cold rooms can be found in (Duret et al., 2014; Hoang et al., 2015; Scaar et al., 2017). A simplified heat transfer modeling in a cold room with loads is presented by (Laguerre et al., 2015). CFD studies of airflow inside cold stores with/without loads, usually accounts local pressure loss source terms, to model fan and cooler unit (Hoang et al., 2000; Nahor et al., 2005; Akdemir and Bartzanas, 2015). In their study, (Wang et al., 2015), simulated the air flow and temperature variation in an ice drink refrigeration cabinet, during an automated dynamic cycle of switching on and switching off. They used a porousjump model for thin perforated plate and pressure drop of 
the fan for air circulation.

The literature review presented above reveals that the detailed numerical study of volume exchange with sliding doors or studies dealing with the infiltration via doors for cold store setup are not abundant in literature. Only a few and very recent works report such attempts, for a cold room (Foster et al., 2003; Carneiro et al., 2017) and for refrigerated vehicles (Zhang et al., 2017). The present study is aimed to perform a numerical analysis by solving compressible form of the conservative 3D laminar NavierStokes equation (without Boussinesq approximation) together with the energy and mass fraction equations for a cold storage room with a product load, a fan, and a sliding door. The commercial computer program StarCCM+ is used for this purpose.

The paper is organized as follows. In section 2 we present the governing equations and methodology for the study of cold storage room. The problem setup is given in section 3 , followed by the results and discussion in section 4. Finally, the conclusions are drawn in section 5.

\section{Method}

\subsection{Governing equations}

The governing equations for the gaseous phase (inside the cold storage room) is the 3D compressible Navier-Stokes system of equations together with mass, species conservation and energy conservation equations. The general transport equation for any conserved property $(\rho \phi)$ can be expressed as in the following standard (Versteeg and Malalasekera, 2007) form:

$$
\frac{\partial \rho \phi}{\partial t}+\vec{\nabla} \cdot \rho \phi \vec{v}=\vec{\nabla} \cdot \Gamma \vec{\nabla} \phi+S_{\phi}
$$

The left hand side consists of the temporal term and the advection term. On the right hand side, the terms signify the diffusion term and the generation term respectively. The solid phase continuum (a block of solid ice as a load in the cold storage room) is governed by the energy conservation equation without any advection or source term. We solve the unsteady Reynolds averaged Navier-Stokes (RANS) formulation. Realizable $\kappa-\varepsilon$ model is used for turbulence. The gravity term in the momentum equations is treated directly without Boussinesq approximation. Newtonian fluid and ideal gas law assumptions serve as the constitutive relation and the equation of state. These close the system of equations with appropriate boundary conditions.

The finite volume method (FVM) based commercial computer program StarCCM+ is used to solve the governing equations in the computational domain. For the present study, the gas continuum consists of two species, namely air and water $\left(\mathrm{H}_{2} \mathrm{O}\right)$ vapor inside the cold storage room. We solve conjugate heat transfer problem by setting the link between the solid phase and the surrounding gaseous medium of the room. The energy and momentum source terms are assigned to represent the effect of the cooler fan in the room without modelling the detail of the cooler unit. The cases with sliding door of the room are handled with the overset mesh setup of StarCCM+. The background mesh (room region) and the overset mesh (door with a surrounding region of choice) are generated for this purpose. A time varying rigid body translation is applied setting the motion parameters associated with the door geometry for the operation of the sliding door opening and closing. Unsteady simulations are performed accordingly. The overall setup of the different test cases is described in section 3. The brief description of the numerical schemes used in the present study is as follows. Second order schemes for convection, hybrid Gauss least-squared gradient method based 2nd order schemes for diffusion and Venkatakrisnan limiter function are chosen in solver setup. First order Euler implicit scheme is used for time integration with a time step $\Delta t=0.1 \mathrm{~s}$. Algebraic Multi-Grid (AMG) techniques are also invoked with the setup mentioned above.

\section{Problem setup}

\subsection{Opened/closed door and sliding door}

A simplified cold storage room with a cooler fan and a sliding door has been considered (see the schematic in figure 1). Table 1 summerizes the Length (L), Breadth (B) and Height $(\mathrm{H})$ of different geometries for the test cases with the fixed door (either fully open or fully closed). The dimension of the door is $1 \mathrm{~m} \times 2 \mathrm{~m}$. All walls of the room are considered as adiabatic. Pressure outlet conditions are set at the door when it is kept open with a prescribed pressure and temperature of the environment. The initial condition of room is set as: temperature, $T=243 \mathrm{~K}$, the mass fraction of air $Y_{\text {air }}=0.9$, the mass fraction of moisture $Y_{\mathrm{H}_{2} \mathrm{O}}=0.1$, velocity field $\vec{v}=0$, pressure $P=0$. The solid ice is kept at: temperature $T=255 \mathrm{~K}$ and noslip wall is satisfied with an appropriate contact interface (with zero resistance) for conjugate heat transfer calculations. At the opened door surface: environment temperaure $T_{\text {env }}=293 \mathrm{~K}, Y_{\text {air }}=0.7, Y_{\mathrm{H}_{2} \mathrm{O}}=0.3, P=0$. The cooler fan specifications are prescribed with an energy source $-100 \mathrm{~W}$, a momentum source $=2 \mathrm{~N} / \mathrm{m}^{3}$ and a momentum source velocity derivative $=2 \mathrm{~kg} / \mathrm{m}^{3} \cdot \mathrm{s}$. 
Table 1. Dimensions for problem setup.

\begin{tabular}{cccccc}
\hline Geometry & $\begin{array}{c}\mathrm{H} \\
(\mathrm{m})\end{array}$ & $\begin{array}{c}\mathrm{L} \\
(\mathrm{m})\end{array}$ & $\begin{array}{c}\mathrm{B} \\
(\mathrm{m})\end{array}$ & $\begin{array}{c}\text { Area } \\
\left(\mathrm{m}^{2}\right)\end{array}$ & $\begin{array}{c}\text { Volume } \\
\left(\mathrm{m}^{3}\right)\end{array}$ \\
\hline Cold room & 2.3 & 3.7 & 3.1 & 11.5 & 26.4 \\
Ice block & 2.0 & 1.0 & 1.0 & 1.0 & 2.0 \\
Cooler fan & 0.3 & 0.5 & 0.3 & 0.15 & 0.045 \\
\hline
\end{tabular}

Table 2. Test cases.

Case 1 Opened door with fan-off

Case 2 Opened door with fan-on

Case 3 Closed door with fan-on

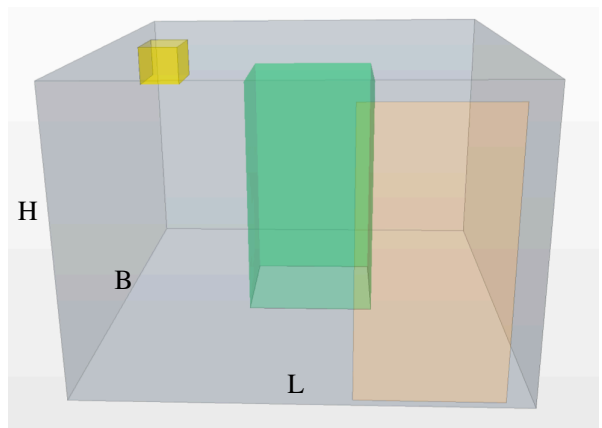

Figure 1. Computational domain of the storage room.

We will first present the results of unsteady simulations with following cases (see table 2) with either closed door or opened door situations (subsection 4.1). The cases with sliding door setup will be presented subsequently in subsection 4.2.

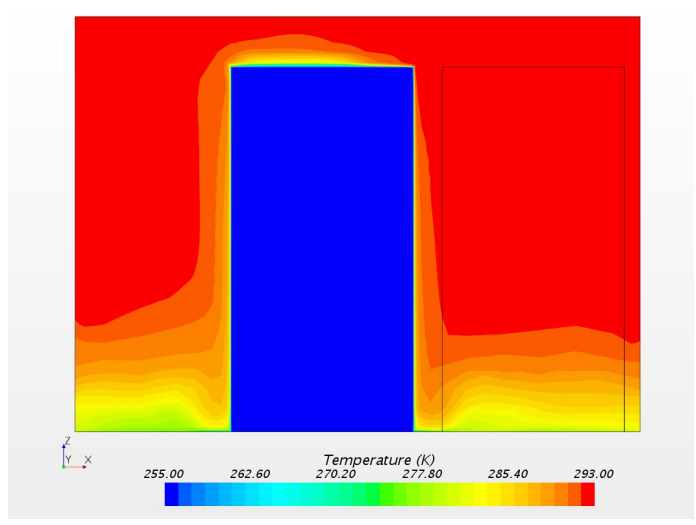

Figure 2. Case 1: contours of $T$ on a $z-x$ plane approximately mid location of the load.

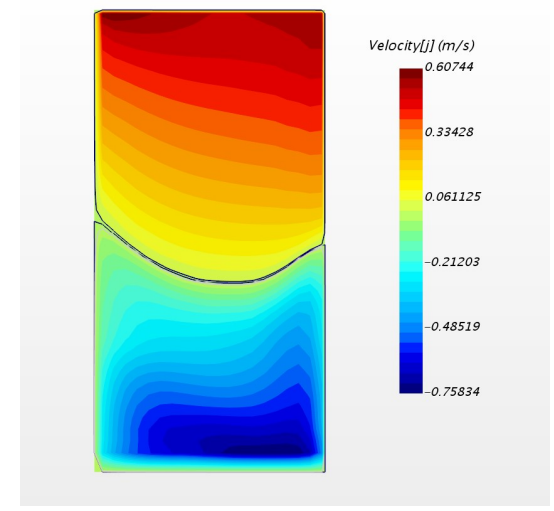

Figure 3. Case 1: contours of $y$-component of velocity through the door. Black curves represent zero velocity.

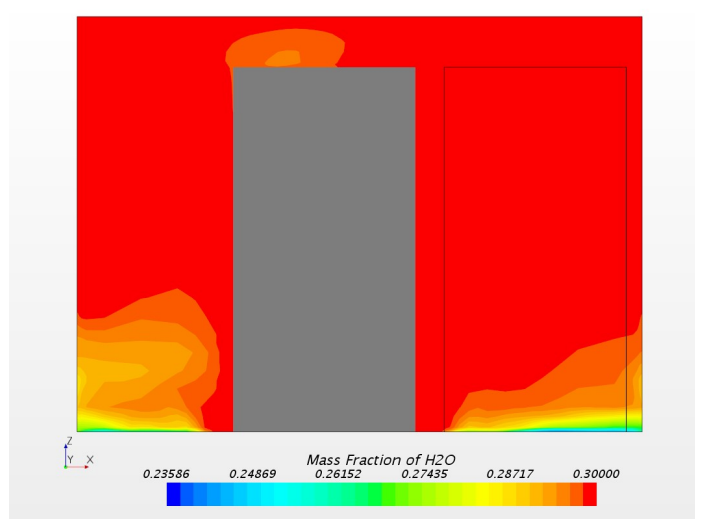

Figure 4. Case 1: contours of $Y_{\mathrm{H}_{2} \mathrm{O}}$.

\section{Results and discussion}

\subsection{Room with fully opened/closed door}

For Case 1, the door is suddenly set as fully opened. The unsteady simulation is performed (till $t=120 \mathrm{~s}$ ) according to the boundary condition mentioned in section 3 . During this time, the air exchange occurred through the door. The room temperature increases as the hot and moist air flows in, through the upper section of the door. Around this time the major portion of the room appears to reach the outdoor temperature (see Fig 2). However, the cold air leaves through the lower section of the door. This is evident from the lower temperature around floor level of the compartment. Figure 3 shows the velocity distribution through the door. The hot air gets cooled as it circulates around the cold product block. From the velocity distribution (see Figure 3), it is clear that the maximum inflow velocity at the upper level of the doorway is lower than the maximum outflow velocity at the bottom level of the doorway. The mass fraction of the water vapor also rapidly gets increased to reach the outdoor condition by this exchange. Figure 4 shows the distribution of the water vapor in the room. The temperature on the surface of the product block is shown in figure 5. As expected, due to the proxi- 


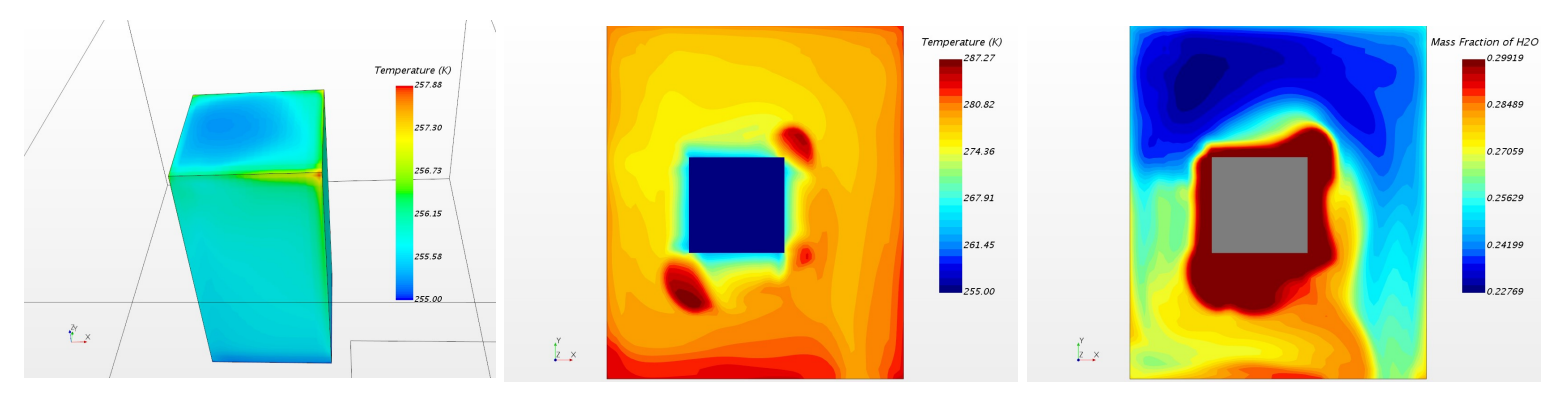

Figure 5. Case 1: $T$ contours at the product surfaces (left), contours of $T$ and $Y_{\mathrm{H}_{2} \mathrm{O}}$ at the floor level (middle and right).
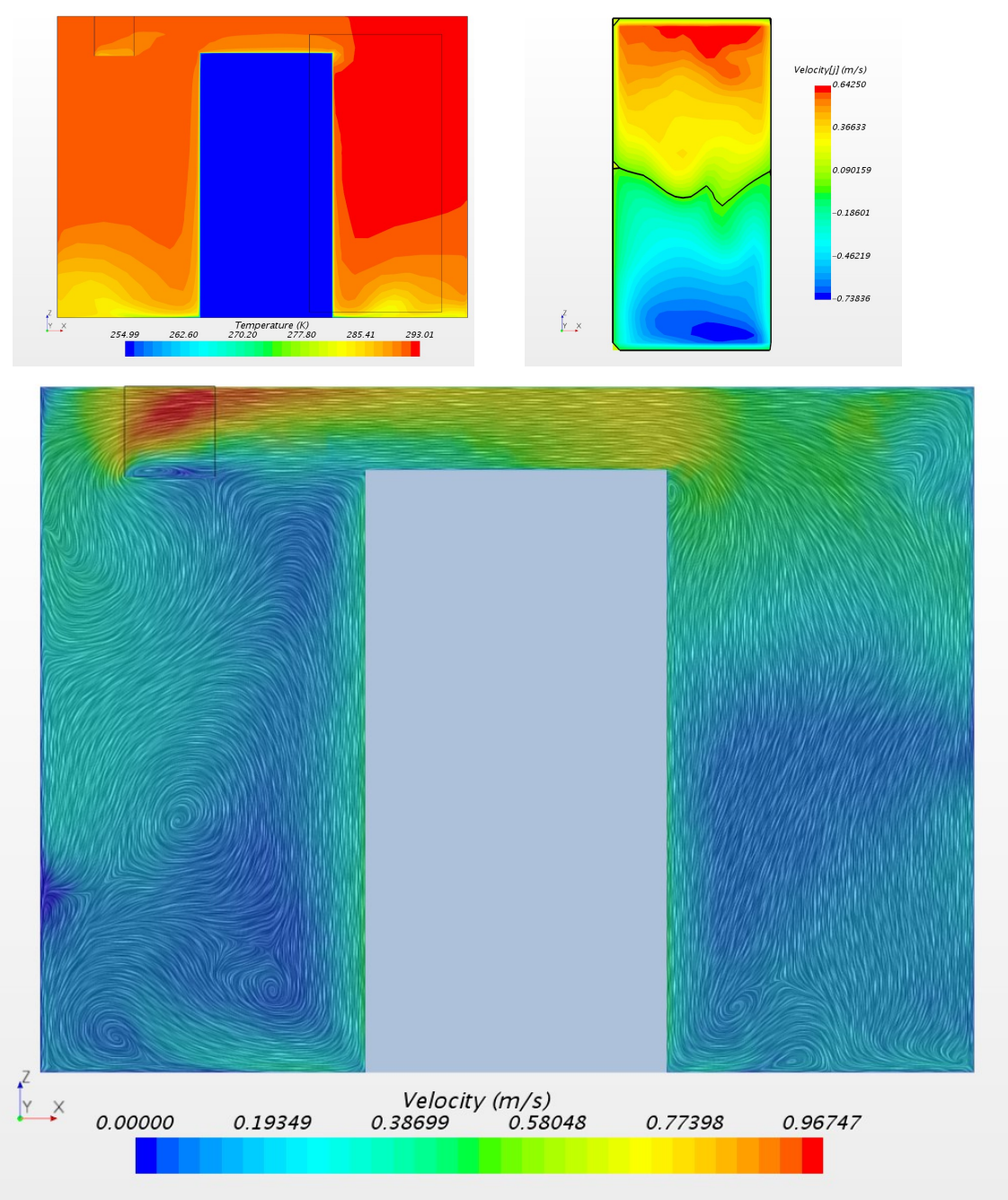

Figure 6. Case 2: contours of $T$ (top left), contours of $y$-component of velocity through the door (top right) and line integral convolution (LIC) of $\vec{v}$ (bottom). Black curves represent zero velocity. 
mity and the exposure to the doorway, the temperature of the nearest surfaces (upper portions) of the product block is found to be higher than the other surfaces. Note that, the temperature of the lower portions of the near-doorway surfaces is lower. This is in accordance with the distribution of temperature in the room as well as near the floor level (see figure 5). Also, note that, the distribution of $Y_{\mathrm{H}_{2} \mathrm{O}}$ reveals the nature of advection and diffusion around the ice block. More and more moist air are dragged along the surfaces of the block downwards due the downward bulk movement of air associtaed with the air exchange process incepted by the opened door.

Case 2 is simulated with similar boundary conditions as Case 1 with the added effect of a cooling fan unit. The energy and momentum source terms are invoked to model the cooler fan as mentioned in section 3. From figure 6 , it can be seen that the a more asymmetric distribution of the temperature field in this plane compared to Case 1. The overall distribution reveals that the temperature of the room increases. The cooler capacity (set by the energy source) is insufficient to cool the room during the $120 \mathrm{se}$ conds time interval. It naturally means the increased cooling load, and can be handled by altering the cooling capacity. For the present case, the airflow induced by the fan actually enhances the mixing of the hot and moist air intake from the opened door. This explains the observed overall temperature distribution for this case. The velocity distribution at the doorway and in the same sample $z-x$ plane is also shown in figure 6. The overall distribution is much different compared to Case 1. This corroborates with the effect of mixing due to the presence of the fan. The fan induced mixed-air flows around the product block also influences the heat transfer through the solid surfaces differently compared to Case 1 . Note that, we observed a net mass flow rate out for both Case 1 and Case 2 through the door at $t=120 \mathrm{~s}$. However, it is found to be $\approx 38 \%$ less mass flow rate out for Case 2 with the cooler fan-on mode compared to Case 1 with fan-off mode.

Figure 7 shows the different contours associated with fan alone when the door is closed (Case 3). The air flow pattern is evidently different compared to the other cases with open door. The cold air circulation from the cooler fan is meant to enhance the freezing of the product load. Figure 7 shows a clear trace of air flow from the fan that contributes to convective hypothermia.

\subsection{Room with sliding door}

In this section, we present the results for a scaled down cold storage room (dimensions are given in table 3) with sliding door of size $6 \mathrm{~cm} \times 12 \mathrm{~cm}$. Figure 8 shows the setup of the computational domain. Here we consider only air as a working fluid. An overset mesh region is built around the sliding door associated with a suitable background mesh. The sliding door is opened and closed with a rigid body motion via a suitable field function of the StarCCM+ program. We solve unsteady laminar Navier-Stokes system of equations for this preliminary setup. For these test ca-
Table 3. Dimensions for sliding door setup.

\begin{tabular}{cccc}
\hline Geometry & $\begin{array}{c}\mathrm{H} \\
(\mathrm{m})\end{array}$ & $\begin{array}{c}\mathrm{L} \\
(\mathrm{m})\end{array}$ & $\begin{array}{c}\mathrm{B} \\
(\mathrm{m})\end{array}$ \\
\hline Cold room & 0.18 & 0.19 & 0.12 \\
Ice block & 0.14 & 0.13 & 0.05 \\
Cooler fan & 0.02 & 0.01 & 0.05 \\
\hline
\end{tabular}

ses, the cold ice block is kept at a constant temperature $(T=255 \mathrm{~K})$ and the energy equation within the ice block is not solved. The fan is modeled in such a way that, it supplies $0.002 \mathrm{~kg} / \mathrm{s}$ of air at a temperature of $T=250 \mathrm{~K}$. The outside temperature is prescribed as $T=300 \mathrm{~K}$. The door is opened during the first $0.8 \mathrm{~s}$ and closed during the next $0.8 \mathrm{~s}$. We present the results at a $55 \%$ open door situation during the opening phase and closing phase of the sliding door. The results are compared for cases with fan-off and fan-on modes. Figure 9 shows the wall normal velocity contours at $55 \%$ open door situations. We found a net mass flow rate out when the fan is off. The net mass flow rates out of the room are almost identical during the opening and closing stages for the fan-off mode. On the other hand, we observed that, the net mass flow rates are different at those stages when the fan is on. The fan induced fluid-flow inside of the room influences the air exchange through the door. During the opening phase, we noticed a net mass flow rate out, while during the closing phase we observed a net mass flow rate in. The net mass flow rate in is about 4.8 times more than the mass flow rate out. Also note that, the net mass flow rate in for the case with the fan-on, is about $42 \%$ less than the net mass flow rate out with fan-off situation. The temperature distributions at a representative plane perpendicular to the door are illustrated in figure 10 and figure 11 . These contours corroborate with the above mentioned air exchange behavior.

\section{Conclusions}

We carried out a numerical study of hot, moist air exchange through a sliding door in a cold storage room. It has been observed that, the energy transport and the cooling of the product load is affected by the fan and the doorway. The air exchange and air mixing patterns differ noticeably in the presence of a fan. The increased demand of the cooling due to the hot and moist air exchange is being noticed. Additionally, a preliminary analysis with transient sliding door setup for a scaled room with one cycle of opening and closing is presented. Without the fan, the air exchange appeared almost identical during the opening or closing phase of the sliding door at $55 \%$ opening. On the other hand, we found a lower air exchange rate with fan-on mode. In future, a detailed study can be opted, taking into account the more realistic condensation of the moist air intake within the framework of a full scale cold storage room with a transient sliding door. 

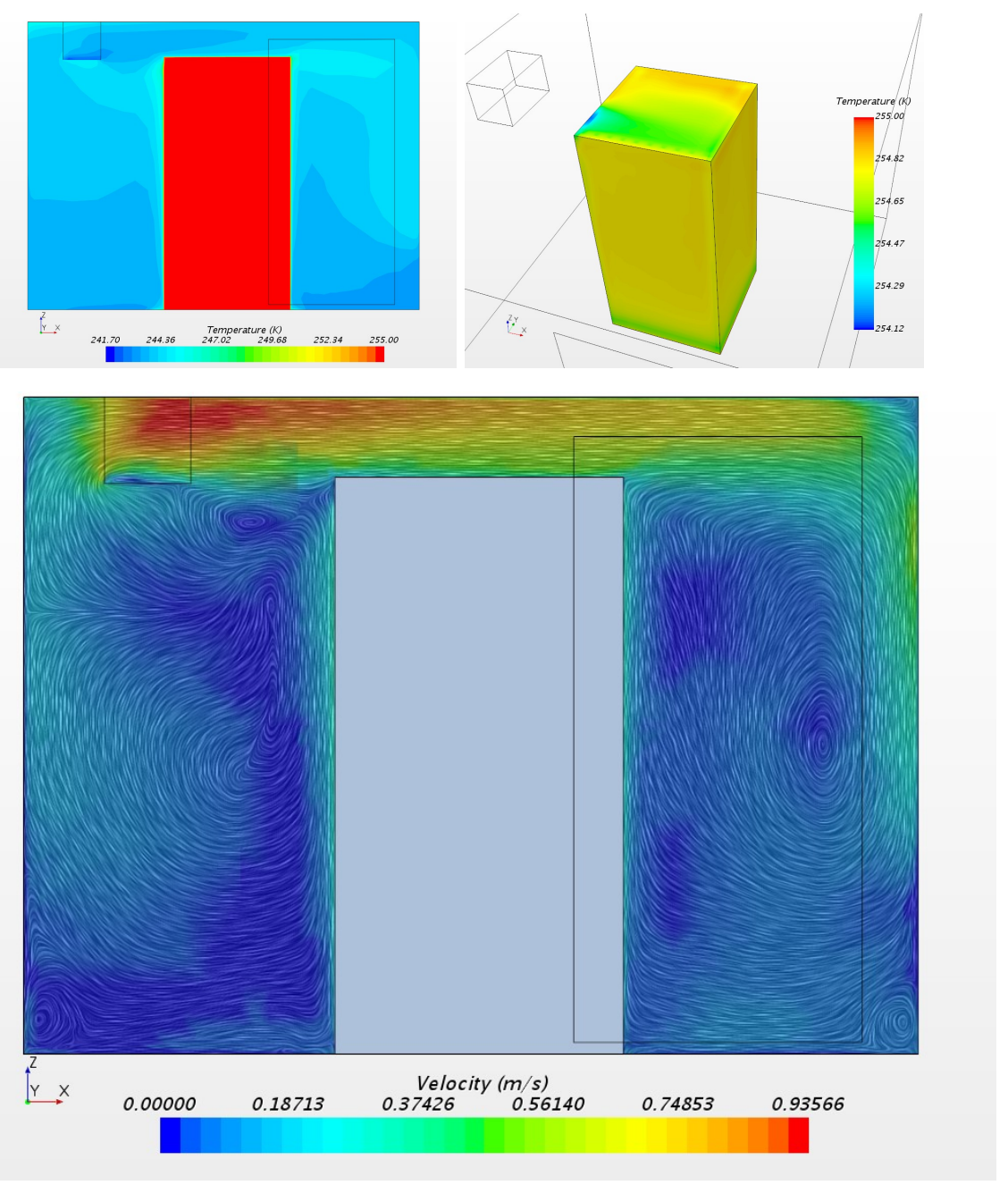

Figure 7. Case 3: contours of $T$ (top left), the $T$ contours (top right) at the product surfaces and LIC of $\vec{v}$ (bottom). 


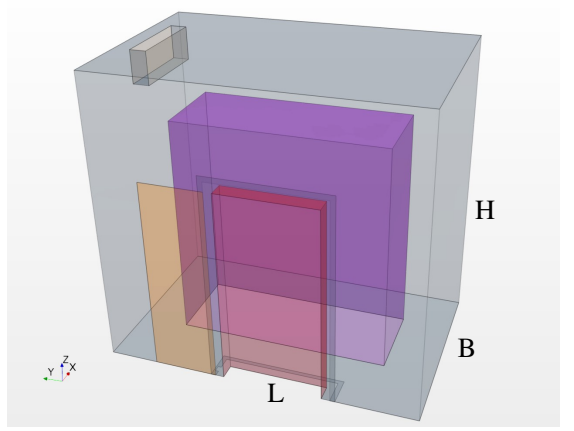

Figure 8. Computational domain of the scaled storage room with sliding door.

\section{Acknowledgement}

Authors greatly acknowledge the commercial computer program StarCCM+, used in this study. The results are obtained by the licensed version 12.06.010 of StarCCM+ on a stand alone desktop at OsloMet - Oslo Metropolitan University.

\section{References}

Serap Akdemir and Thomas Bartzanas. Numerical modelling and experimental validation of a cold store. Tarım Bilimleri Dergisi, 21(4):606-619, 2015.

Rui Carneiro, PD Gaspar, and PD Silva. 3d and transient numerical modelling of door opening and closing processes and its influence on thermal performance of cold rooms. Applied Thermal Engineering, 113:585-600, 2017.

Le Chang, Xu Zhang, Shengji Wang, and Jun Gao. Control room contaminant inleakage produced by door opening and closing: Dynamic simulation and experiments. Building and Environment, 98:11-20, 2016.

J-I Choi and Jack R Edwards. Large-eddy simulation of humaninduced contaminant transport in room compartments. Indoor air, 22(1):77-87, 2012.

S Duret, H-M Hoang, Denis Flick, and O Laguerre. Experimental characterization of airflow, heat and mass transfer in a cold room filled with food products. International journal of refrigeration, 46:17-25, 2014.

AM Foster, MJ Swain, R Barrett, and SJ James. Experimental verification of analytical and cfd predictions of infiltration through cold store entrances. International Journal of Refrigeration, 26(8):918-925, 2003.

Hong-Minh Hoang, Steven Duret, Denis Flick, and Onrawee Laguerre. Preliminary study of airflow and heat transfer in a cold room filled with apple pallets: Comparison between two modelling approaches and experimental results. Applied Thermal Engineering, 76:367-381, 2015.

My Lan Hoang, Pieter Verboven, Josse De Baerdemaeker, and BM Nicola1. Analysis of the air flow in a cold store by means of computational fluid dynamics. International Journal of Refrigeration, 23(2):127-140, 2000.
O Laguerre, S Duret, HM Hoang, Laurent Guillier, and Denis Flick. Simplified heat transfer modeling in a cold room filled with food products. Journal of Food Engineering, 149:78-86, 2015.

HB Nahor, ML Hoang, Pieter Verboven, Martine Baelmans, and BM Nicolai. Cfd model of the airflow, heat and mass transfer in cool stores. International Journal of Refrigeration, 28(3): 368-380, 2005.

Ilias G Papakonstantis, Elizabeth Abigail Hathway, and Wernher Brevis. An experimental study of the flow induced by the motion of a hinged door separating two rooms. Building and Environment, 2017.

Holger Scaar, Ulrike Praeger, Klaus Gottschalk, Reiner Jedermann, and Martin Geyer. Experimentelle und numerische analyse der luftströmung in obst-und gemüselagern. LANDTECHNIK-Agricultural Engineering, 72(1), 2017.

Julian W Tang, Andre Nicolle, Jovan Pantelic, Christian A Klettner, Ruikun Su, Petri Kalliomaki, Pekka Saarinen, Hannu Koskela, Kari Reijula, Panu Mustakallio, et al. Different types of door-opening motions as contributing factors to containment failures in hospital isolation rooms. PloS one, 8(6): e66663, 2013.

JW Tang, I Eames, Y Li, YA Taha, P Wilson, G Bellingan, $\mathrm{KN}$ Ward, and J Breuer. Door-opening motion can potentially lead to a transient breakdown in negative-pressure isolation conditions: the importance of vorticity and buoyancy airflows. Journal of Hospital Infection, 61(4):283-286, 2005.

Henk Kaarle Versteeg and Weeratunge Malalasekera. An introduction to computational fluid dynamics: the finite volume method. Pearson Education, 2007.

Limin Wang, Lin Zhang, and Guoping Lian. A cfd simulation of $3 \mathrm{~d}$ air flow and temperature variation in refrigeration cabinet. Procedia engineering, 102:1599-1611, 2015.

DJ Wilson and DE Kiel. Gravity driven counterflow through an open door in a sealed room. Building and Environment, 25 (4):379-388, 1990.

Xiang Zhang, Jia-Wei Han, Jian-Ping Qian, Yi-Zhong Wang, Lin Wang, and Xin-Ting Yang. Computational fluid dynamic study of thermal effects of open doors of refrigerated vehicles. Journal of Food Process Engineering, 2017. 

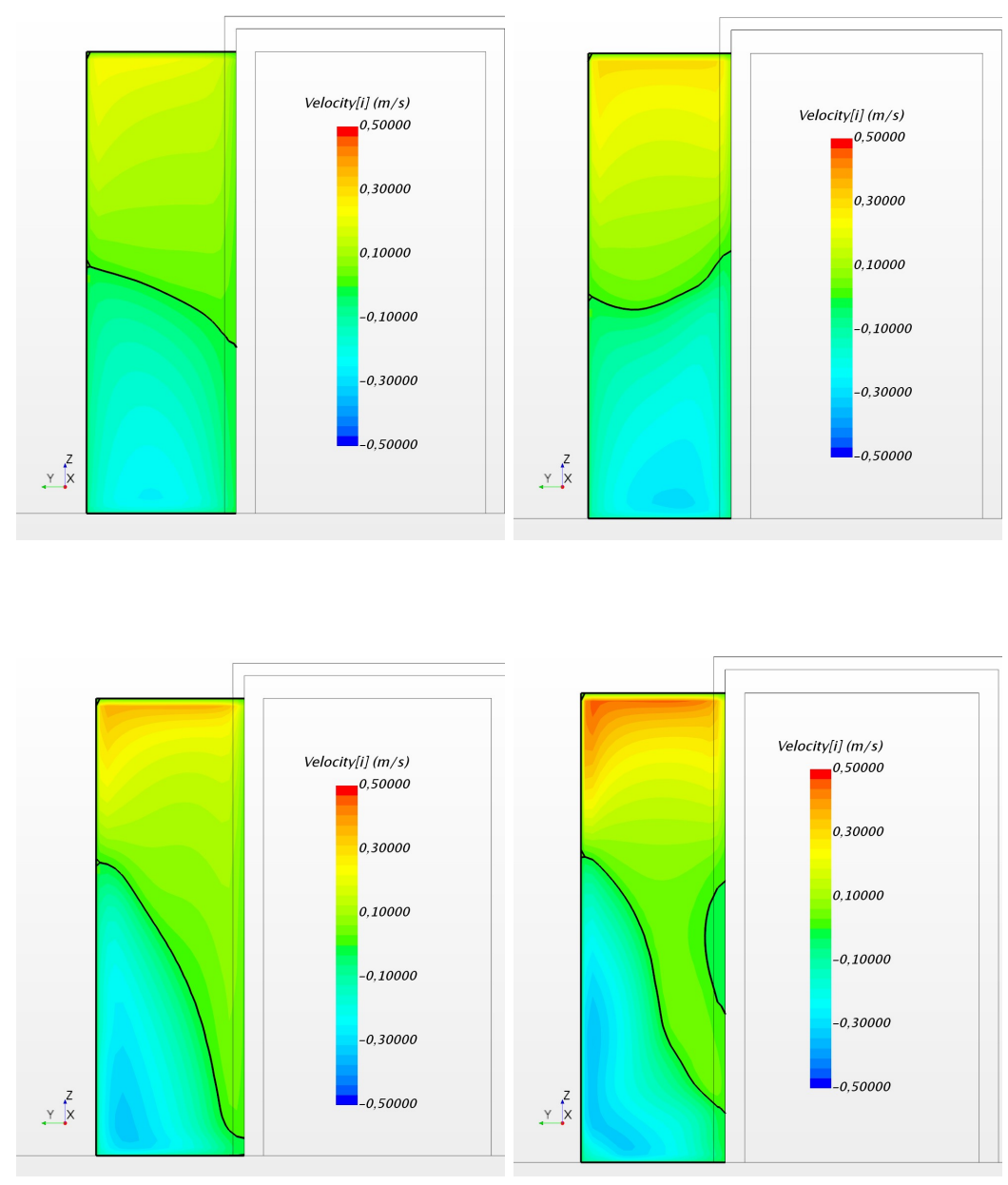

Figure 9. Door normal velocity contours at $t=0.44 \mathrm{~s}$ (left column) and at $t=1.16 \mathrm{~s}$ (right column). Top row: with fan-off mode, bottom row: with fan-on mode. Black curves represent zero velocity.

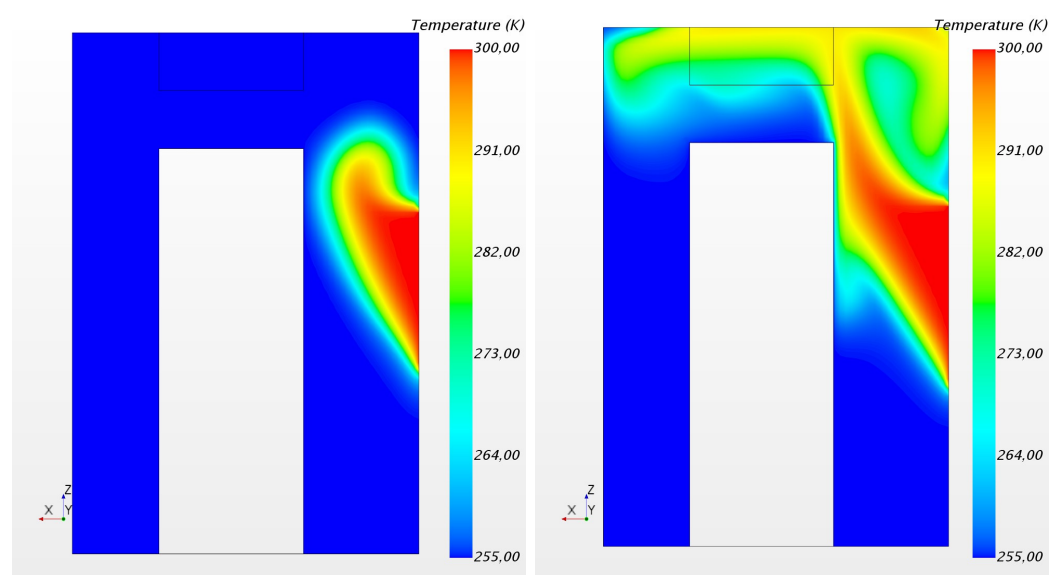

Figure 10. Temperature contours in a representative plane perpendicular to the door at $t=0.44 \mathrm{~s}$ (left) and at $t=1.16 \mathrm{~s}$ (right) with fan-off mode. 


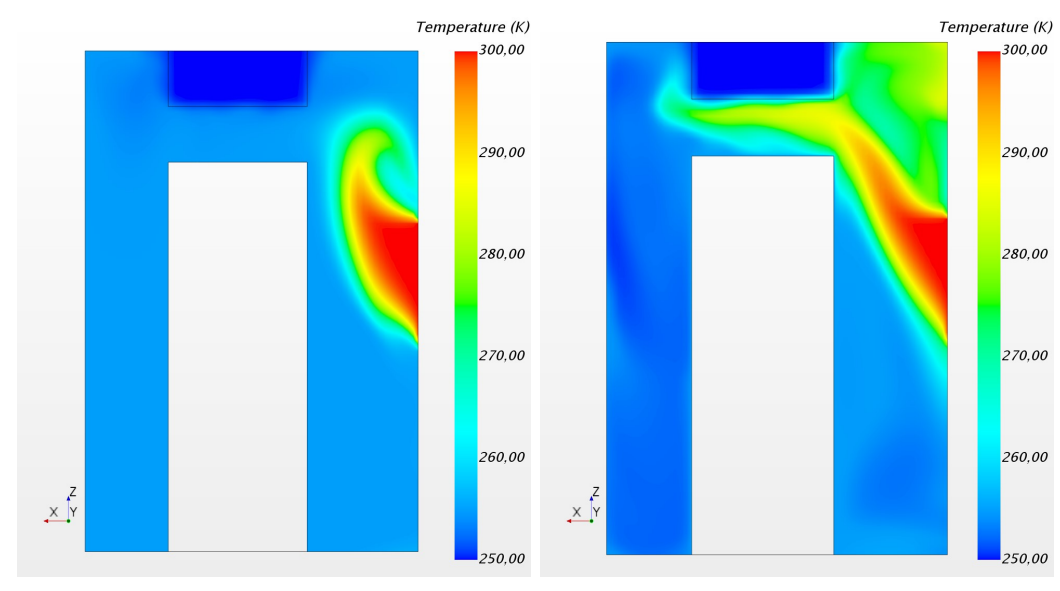

Figure 11. Temperature contours in a representative plane perpendicular to the door at $t=0.44 \mathrm{~s}$ (left) and at $t=1.16 \mathrm{~s}$ (right) with fan-on mode. 